\title{
Evaluation of Contraception Access within the Indianapolis Metropolitan Area
}

Grace M. Yedlicka ${ }^{1}$, Emily R. Theis ${ }^{2}$, Caitlin Bernard ${ }^{2}$, Brownsyne Tucker Edmonds ${ }^{2}$, Jeffrey F. Peipert $^{2}$, Tracey A. Wilkinson ${ }^{3}$

${ }^{1}$ Indiana University School of Medicine; ${ }^{2}$ Indiana University School of Medicine, Department of Obstetrics and Gynecology; ${ }^{3}$ Indiana University School of Medicine, Department of Pediatrics, Children's Health Services Research

Background: Access to family planning care and the full-spectrum of contraception, including same-day access to long acting reversible contraception (LARC) is critical to reduce unintended pregnancy and maternal/infant mortality. However, access remains variable for many women in Indiana. We sought to examine access to various contraceptive practices within the Indianapolis metropolitan area.

Methods: A telephone survey of clinic managers was conducted between June-July 2020 of all identified IU Health, Eskenazi, and HealthNet primary care and OB/GYN providers in the Indianapolis metropolitan area. The 27-question survey gathered information regarding contraceptive practices (e.g. contraceptive method availability, same-day LARC insertion/removal, pregnancy intention screening, educational materials, and parental consent policies). The data was compared based on type of medical provider (OB/GYN, Family Medicine, and Pediatrics).

Results: In total, 82 sites were identified, 58 surveys $(70.7 \%)$ were completed. Oral contraception is the most commonly offered contraception $(91.4 \%)$, followed by depot medroxyprogesterone acetate (DMPA) injection (84.5\%), and the contraceptive patch (81.0\%). LARC placement is available in $51.7 \%$ of all clinics, but only $34.5 \%$ offer same-day placements. Routine pregnancy intention screening is offered in $43.1 \%$ and educational materials in $74.1 \%$ of practices. OB/GYN clinics provide the most comprehensive access, $69.2 \%$ provide same-day LARC insertion (84.6\% LARC removals). Family medicine practices are variable regarding contraceptive choices: over $91 \%$ offer oral contraception and DMPA injections, but only $20.8 \%$ place LARCs and $41.7 \%$ remove LARCs. Half $(50 \%)$ of pediatric clinics provide oral contraception, while $50 \%$ do not provide any contraceptives. Parental consent for minors to obtain contraception provision is highly variable.

Conclusion: Contraceptive practices vary among primary care and OB/GYN providers in the Indianapolis area. This variability in access leads to barriers for use, particularly for young women. Efforts to ensure comprehensive, same-day access to all forms of contraception (including LARCs), pregnancy intention screening, and contraceptive educational materials are needed. 